\title{
Stochastic Replay of SIMO Underwater Acoustic Communication Channels
}

\author{
Francois-Xavier Socheleau, Antony Pottier, Christophe Laot \\ Institut Mines-Telecom; TELECOM Bretagne, UMR CNRS 6285 Lab-STICC \\ Email: \{fx.socheleau, antony.pottier,christophe.laot\}@telecom-bretagne.eu
}

\begin{abstract}
Stochastic replay is a channel simulation method that generates random time-varying impulse responses (TVIRs) from data collected at sea. Most existing replay-based simulators only consider single-input single-output transmissions. In this paper, a single-input multiple-output (SIMO) stochastic replaybased simulator is presented. It is shown to keep temporal, inter-tap as well as spatial correlations consistent with those of the TVIR measured at sea. Numerical examples applied to data collected in Brest harbor, France, are discussed.
\end{abstract}

Index Terms-Channel simulation, underwater acoustic communications, SIMO, spatial correlation

\section{INTRODUCTION}

B Ecause of their high degree of realism, replay-based channel simulators are steadily getting more attention in underwater acoustic communications [1]-[7]. Driven by a time-varying impulse response (TVIR) measured in situ during at-sea experiments, these simulators are able to generate new random TVIRs with statistical properties (almost) identical to the original measurement. Although scenario-specific, replaybased simulators are very relevant to perform fair comparisons of underwater acoustic communication (UAC) protocols.

So far, existing replay-based strategies have only focused on single-input single-output channels. In practice, UAC systems often use several hydrophones at reception so as to benefit from receive diversity in order to combat fading and increase signal-to-noise ratio (SNR). Therefore, extension of replaybased simulators to single-input multiple-output (SIMO) configurations is needed.

Most SIMO UAC channels exhibit time variation, multipath delay spread and spatial selectivity [8]-[10]. Such channels are said to be triply selective and may undergo temporal, intertap as well as spatial correlations. Consequently, the main challenge faced by SIMO replay-based simulators is to keep these correlations consistent with those of the TVIR measured at sea.

In this paper, we show that all these correlations can be jointly preserved by a simple SIMO replay method that relies on a randomized Fourier transform of the channel taps. More precisely, in Sec. II we present the trend stationary Rician fading model which our simulator is based on. The SIMO replay strategy is detailed in Sec. III. Real data measured in Brest harbor, France, are studied in Sec. IV. It is shown that intertap as well as spatial correlations do exist in practice, even for a hydrophone spacing of more than 20 wavelengths. Some applications of the proposed SIMO replay simulator are also shown in this section. Finally, conclusions and perspectives are discussed in Sec. V.

Notations: Throughout this paper, uppercase boldface letters denote matrices, e.g., A. The superscripts ${ }^{T}$ and ${ }^{\dagger}$ denote transposition and Hermitian transposition, respectively. The entries of a matrix $\mathbf{A}$ are denoted by $[\mathbf{A}]_{k, n} . \mathbf{I}_{N}$ designates the $N \times N$ identity matrix. $[\cdot]_{K}$ is the modulo $K$ operator. Finally, $\mathbb{E}\{$.$\} denotes expectation.$

\section{ChANNEL MODEL}

We consider a SIMO underwater acoustic channel, modeled as a random linear time-varying system $\mathbb{H}$ that maps an input signal $x(t)$ onto $M$ output signals $z^{(m)}(t), m \in\{1, \cdots, M\}$, according to the $\mathrm{I} / \mathrm{O}$ relationship

$$
z^{(m)}(t)=\int_{\tau} h_{\mathbb{H}}^{(m)}(\tau, t) x(t-\tau) d \tau+w^{(m)}(t),
$$

where $h_{\mathbb{H}}^{(m)}(\tau, t)$ is the channel impulse response between the transmitter and the $m$-th hydrophone and $w^{(m)}(t)$ denotes the ambient noise. As common practice in replay-based simulation [2]-[4], the input of the simulator is a discrete-time baseband estimate of the in-situ TVIR whose time-varying Doppler shift has been removed. Such an estimate is denoted as $h_{l}^{(m)}(k)$ where $l \in\{0, \cdots, L-1\}$ is the tap index and $k \in\{0, \cdots, K-1\}$ is the time index. The removal of the Doppler shift is required to mitigate the drift of multipath arrivals that could obscure the analysis of the taps statistics. Apparent Doppler shift occurs because of relative motion as well as clock-frequency mismatch between the transmitter and the receiver. Once the channel statistical properties have been analyzed, this Doppler shift can be reinserted at the output of the simulator by performing time resampling. The channel estimation method as well as the Doppler removal procedure are described in Sec. IV.

In agreement with [3], each impulse response is modeled as a multi-variate trend stationary random process ${ }^{1}$ so that, for all $k, k_{1}$ and $k_{2} \in \mathbb{Z}$

$$
h_{l}^{(m)}(k)=\bar{h}_{l}^{(m)}(k)+\tilde{h}_{l}^{(m)}(k),
$$

with

$$
\mathbb{E}\left\{h_{l}^{(m)}(k)\right\}=\bar{h}_{l}^{(m)}(k),
$$

${ }^{1}$ For simplicity, we use the same notation to denote a random process and one of its possible sample path. It is also assumed that the pseudocovariances $\mathbb{E}\left\{\tilde{h}_{l}^{(m)}\left(k_{1}\right) \tilde{h}_{p}^{(n)}\left(k_{2}\right)\right\}$ are negligible. 


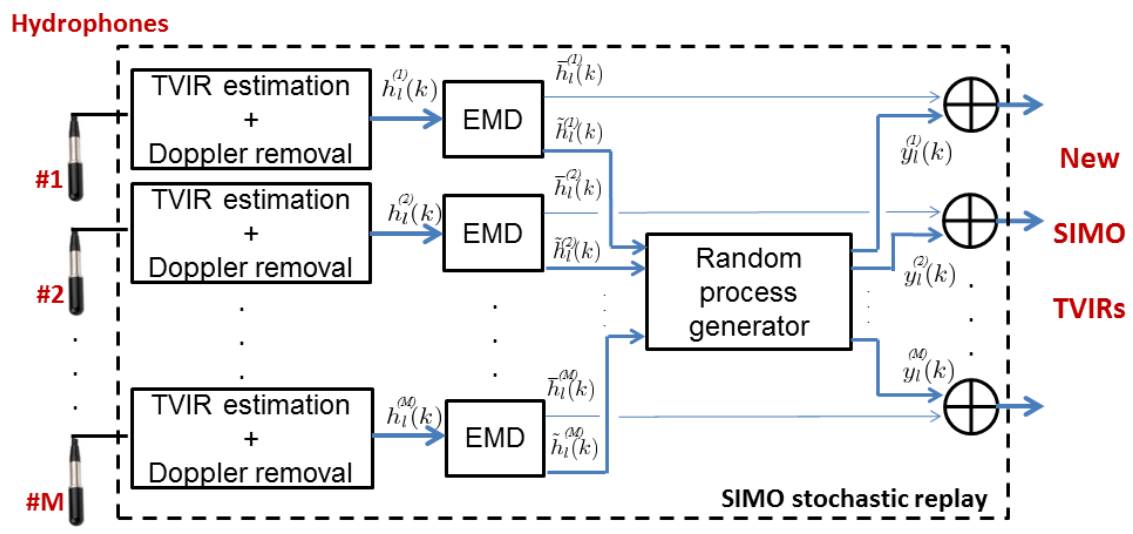

Fig. 1. SIMO stochastic replay simulator.

and

$\mathbb{E}\left\{\tilde{h}_{l}^{(m)}\left(k_{1}\right) \tilde{h}_{p}^{*(n)}\left(k_{2}\right)\right\}=\mathbb{E}\left\{\tilde{h}_{l}^{(m)}(k) \tilde{h}_{p}^{*(n)}\left(k+k_{2}-k_{1}\right)\right\}$. $\bar{h}_{l}^{(m)}(k)$ is called the trend and is a slowly time-varying deterministic component. $\tilde{h}_{l}^{(m)}(k)$ is a zero-mean wide-sense stationary random process assumed to be Gaussian. This model describes the UA channel as a multivariate Rician fading process with a slowly time-varying mean. $\bar{h}_{l}^{(m)}(k)$ can be interpreted as the contribution of (pseudo) deterministic physical phenomena to channel fluctuations, i.e. $\bar{h}_{l}^{(m)}(k)$ is a (pseudo) specular component, and $\tilde{h}_{l}^{(m)}(k)$ represents the channel fluctuations attributable to scatterers that result in fast fading.

\section{SIMO STOCHASTIC REPLAY}

Based on (2), standard replay methods first isolate both components $\bar{h}_{l}^{(m)}(k)$ and $\tilde{h}_{l}^{(m)}(k)$ from $h_{l}^{(m)}(k)$. For each TVIR, this is done by estimating the specular component $\bar{h}_{l}^{(m)}(k)$ using an empirical mode decomposition (EMD) as detailed in [3]. $\tilde{h}_{l}^{(m)}(k)$ is then obtained as the difference between the observation $h_{l}^{(m)}(k)$ and the estimated $\bar{h}_{l}^{(m)}(k)$. Illustrations of EMD outputs are shown in [11, Fig. 2], [3, Fig. 7] and [5, Fig. 1]. The SIMO stochastic replay simulator generate new realizations of the channel random components based on the observations $\tilde{h}_{l}^{(m)}(k)$. These realizations are then added to the original specular components $\bar{h}_{l}^{(m)}(k)$ to obtain new TVIRs. The overall SIMO replay-based simulation methodology is shown in Figure 1.

The aim of our replay method is to generate new realizations of the channel taps that keep the same statistics as the measured TVIRs. Formally, we seek to generate sample paths of a Gaussian multivariate random process that has a covariance matrix identical to

$$
\boldsymbol{\Sigma}_{\tilde{h}}(u) \triangleq\left[\begin{array}{cccc}
\boldsymbol{\Sigma}_{\tilde{h}}^{(1,1)}(u) & \boldsymbol{\Sigma}_{\tilde{h}}^{(1,2)}(u) & \ldots & \boldsymbol{\Sigma}_{\tilde{h}}^{(1, M)}(u) \\
\boldsymbol{\Sigma}_{\tilde{h}}^{(2,1)}(u) & \boldsymbol{\Sigma}_{\tilde{h}}^{(2,2)}(u) & \ddots & \vdots \\
\vdots & \vdots & \ddots & \vdots \\
\boldsymbol{\Sigma}_{\tilde{h}}^{(M, 1)}(u) & \ldots & \ldots & \boldsymbol{\Sigma}_{\tilde{h}}^{(M, M)}(u)
\end{array}\right] \text {, }
$$

where the matrix sub-block $\Sigma_{\tilde{h}}^{(m, n)}(u)$ of size $L \times L$ satisfies

$$
\left[\boldsymbol{\Sigma}_{\tilde{h}}^{(m, n)}(u)\right]_{l p} \triangleq \mathbb{E}\left\{\tilde{h}_{l}^{(m)}(k) \tilde{h}_{p}^{*(n)}(k+u)\right\} .
$$

This matrix represents the channel tap covariance jointly in time, delay and space. The TVIRs are assumed to be ergodic so that the entries of the covariance matrix also satisfy

$$
\left[\boldsymbol{\Sigma}_{\tilde{h}}^{(m, n)}(u)\right]_{l p}=\lim _{K \rightarrow+\infty} \frac{1}{2 K+1} \sum_{k=-K}^{+K} \tilde{h}_{l}^{(m)}(k) \tilde{h}_{p}^{*}(n)(k+u) .
$$

Following the same approach as in [3], we propose to simulate new sample paths of the random components as follows

$$
y_{l}^{(m)}(k)=\frac{1}{K} \sum_{v=0}^{K-1} \tilde{H}_{l}^{(m)}(v) e^{2 i \pi v k / K} e^{i \theta_{l}^{(m)}(v)},
$$

where $\tilde{H}_{l}^{(m)}(v)=\sum_{k=0}^{K-1} \tilde{h}_{l}^{(m)}(k) e^{-2 i \pi v k / K}$, and $\theta_{l}^{(m)}(v)$ is a random phase uniformly distributed in $(0,2 \pi]$. To keep similar statistics as the measured TVIRs, this random phase must satisfies

$\left\{\begin{array}{cc}\theta_{l}^{(m)}(v)=\theta_{p}^{(n)}(v), & \forall l, p, m, n, \\ \theta_{l}^{(m)}(v) \text { and } \theta_{l}^{(m)}(w) & \text { mutually independent for } v \neq w .\end{array}\right.$

In that case, the covariance of $y$ satisfies, for $\frac{-K}{2} \leq u \leq \frac{K}{2}$,

$$
\begin{aligned}
{\left[\boldsymbol{\Sigma}_{y}^{(m, n)}(u)\right]_{l p} } & \stackrel{(a)}{=} \mathbb{E}_{\tilde{h}}\left\{\mathbb{E}_{\theta}\left\{y_{l}^{(m)}(k) y_{p}^{*(n)}(k+u) \mid \tilde{h}\right\}\right\} \\
& \stackrel{(b)}{=} \mathbb{E}_{\tilde{h}}\left\{\frac{1}{K} \sum_{k=0}^{K-1} \tilde{h}_{l}^{(m)}(k) \tilde{h}_{p}^{*(n)}\left([k+u]_{K}\right)\right\} \\
& \stackrel{(c)}{\approx} \frac{K-|u|}{K}\left[\boldsymbol{\Sigma}_{\tilde{h}}^{(m, n)}(u)\right]_{l p}
\end{aligned}
$$

where (a) follows from the law of iterated expectation and (b) from (8) and Parseval's theorem. (c) is obtained by making the assumption that the covariance is negligible for $|u|>\frac{K}{2}$. This assumption is valid as soon as the duration of the measured TVIRs is longer than the coherence time of their random components. For the experiment described in Sec . IV, the TVIRs were measured over a period of $30 \mathrm{~s}$., which is long compared to a coherence time that is of a few hundreds of ms. Note that even in this case, the covariance of $y$ expressed in (9) is similar to the one of $\tilde{h}$ but up to the scale factor $(K-|u|) / K$. Once again, when $K$ is large this is not a major concern since, in general, the entries of $\boldsymbol{\Sigma}_{\tilde{h}}(u)$ rapidly decrease with $u$. 


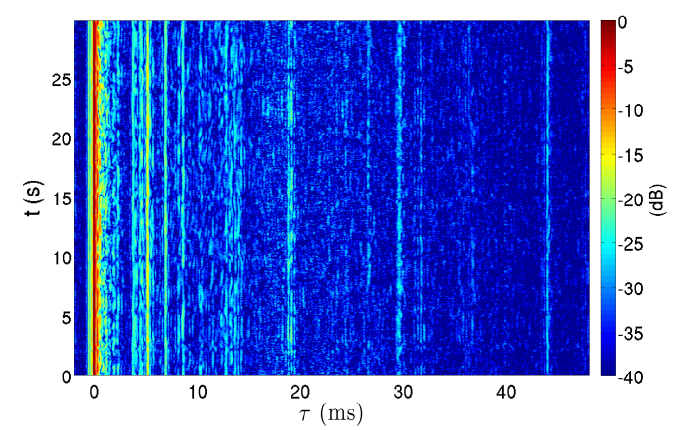

(a) Channel \#1

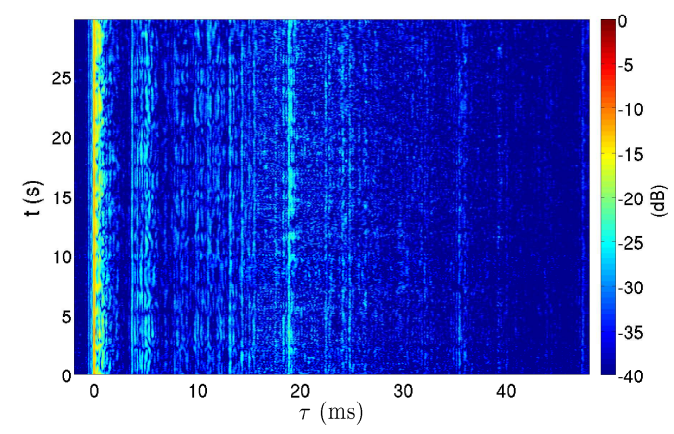

(c) Channel \#3

Fig. 2. Magnitude of $h_{l}^{(m)}(k), m=1,2,3,4$.

\section{ILLUSTRATIONS}

\section{A. Experiment set-up}

The SIMO replay-based simulation strategy is here illustrated with real data collected in Brest harbor, France. Channels measurements were conducted in this harbor in May 2015. The transmissions were realized between two docks over a $800 \mathrm{~m}$ distance, in a $20 \mathrm{~m}$ water depth. At the transmitter side, an omnidirectional transducer ITC-1032 (resonant frequency: $35 \mathrm{kHz}$ ) immersed at a depth of $2 \mathrm{~m}$ was used. At the receiver side, four broadband omnidirectional BK8106 hydrophones were vertically deployed at a depth between 3 and $6 \mathrm{~m}$ with a $1 \mathrm{~m}$ spacing. Estimates of the TVIRs were obtained by successive matched filtering to a known probe signal transmitted repeatedly. The probe signal used during the experiments was a m-sequence of 255 BPSK chips transmitted at a symbol rate of $5 \mathrm{kbds}$. Such a sequence can capture arrivals delayed up to $51 \mathrm{~ms}$ and channel estimates can be updated up to 19.6 times per second. Measurements were made at a carrier frequency of $35 \mathrm{kHz}$ and transmitted symbols were pulse-shaped by a root raised cosine filter with a roll-off factor of 0.1 .

At the receiver side, the recorded signal is first downconverted into complex-baseband and downsampled. Then, time-varying Doppler shifts are mitigated by an iterative resampling procedure presented in [2]: the first step consists in the elimination of a coarse, constant Doppler shift estimated by a bank of Doppler-shifted replicas of the transmitted probe signal. Following this step, a first estimate of the TVIR can be obtained to compute the center of gravity of its Doppler power spectrum, from which a finer resampling factor can be

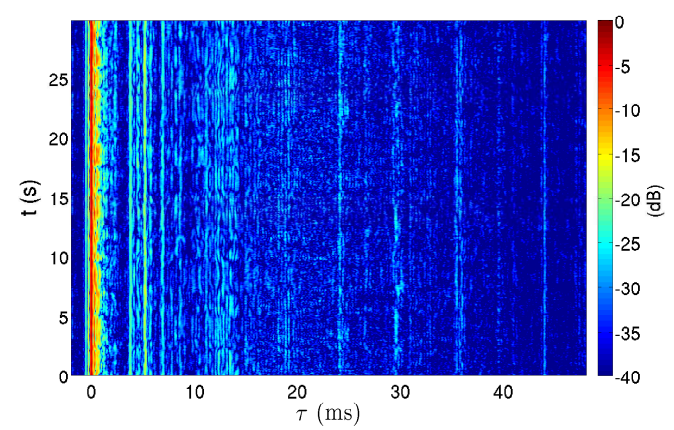

(b) Channel \#2

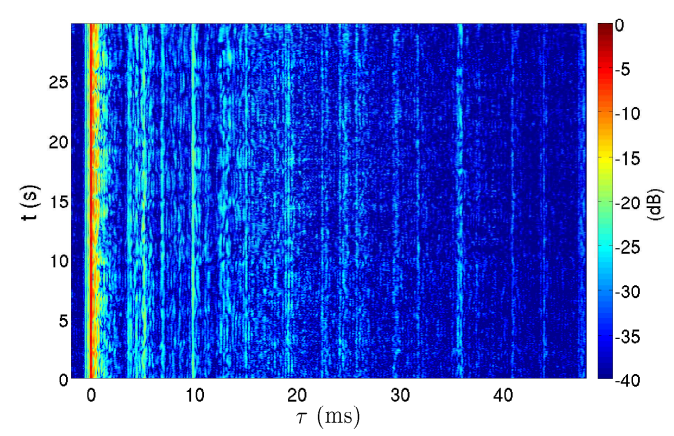

(d) Channel \#4

deduced. In our experiments, these two steps mainly compensate clock frequency offsets of electronic devices. A last correction is applied to remove time-varying Doppler shifts around the mean. To this end, a time-varying resampling factor is computed from the unwrapped phase of the most energetic arrival.

Fig. 2 shows the channel estimates obtained on the four hydrophones. The strongest arrival is arbitrarily placed at zero delay. These four TVIRS present some similarities. For all of them, the main tap, which corresponds the direct path, is very stable and the rms delay spread is approximately $6.5 \mathrm{~ms}$. However, energetic differences can be noticed. For instance, the total energy received at the third hydrophone is $8 \mathrm{~dB}$ lower than at the first hydrophone. In addition, the amount of energy carried by the specular components $\bar{h}_{l}^{(m)}(k)$ slightly varies from one channel to another. It represents about $75 \%$ for channel \#1, whereas it is less than $50 \%$ for channel \#3.

Despite a relative hydrophone spacing corresponding to more than 20 wavelengths, the four TVIRs are highly correlated. This is due in large part of the substantial amount of specular energy but not only. As shown in Fig. 3, some inter-tap and spatial correlations also exist between the random components. This observation reveals that care must be taken when invoking the common assumption of uncorrelated scattering or when applying rules of thumb on the relationship between the wavelength and the inter-channel correlation.

\section{B. Stochastic replay of real data}

To show that stochastic replay actually keeps the intertap and spatial correlations of the in situ channel, population correlation matrices are displayed in Fig. 4. While the 

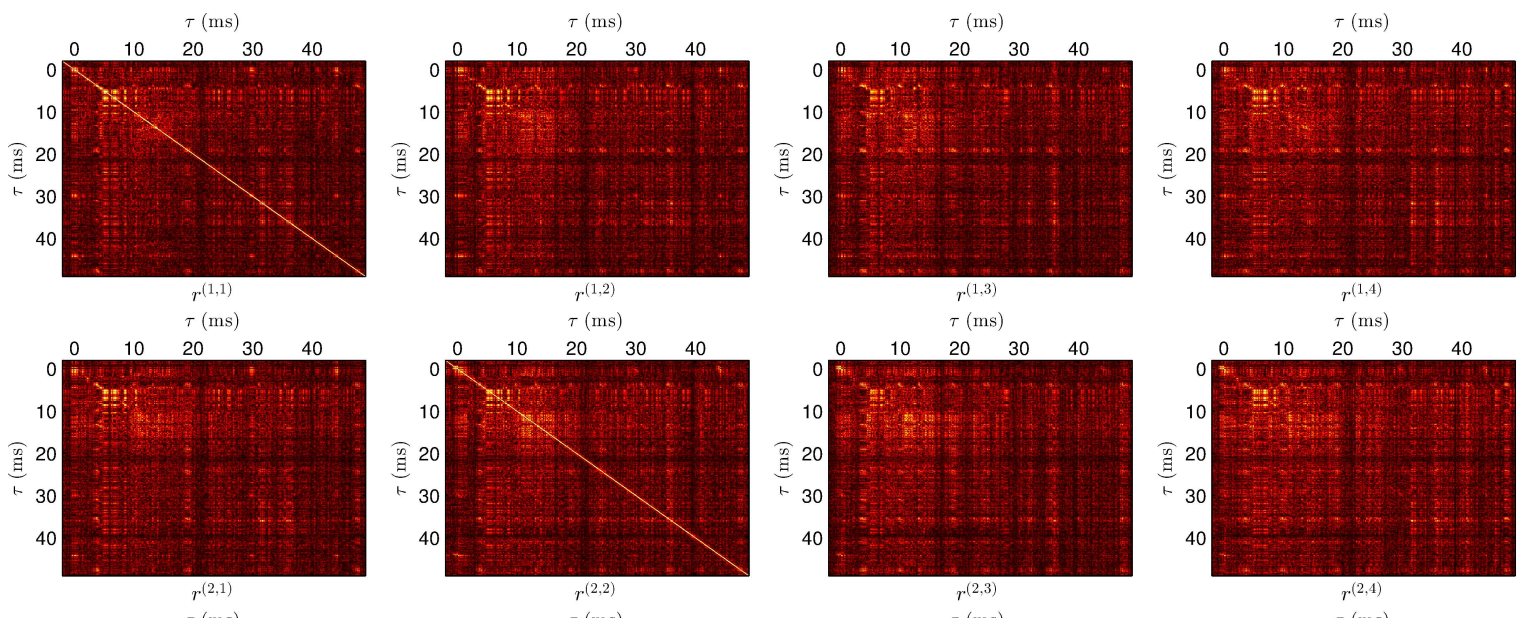

$\tau(\mathrm{ms})$
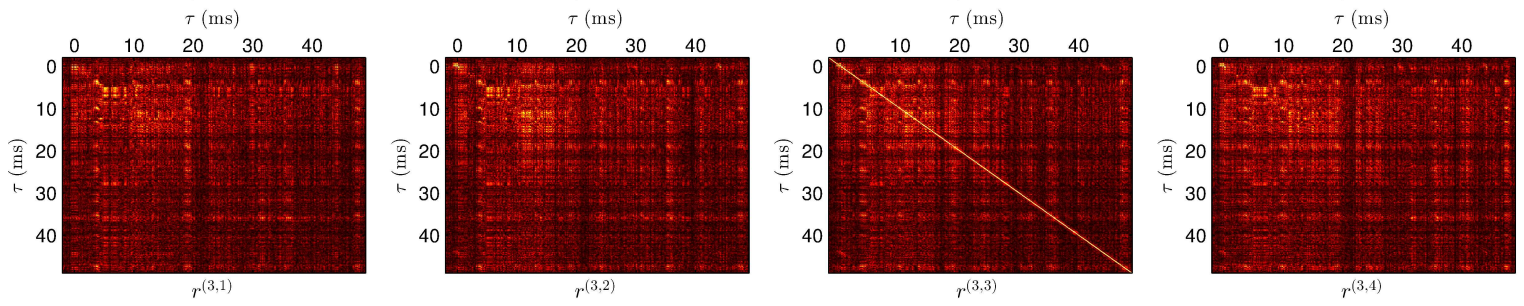

$\tau(\mathrm{ms})$
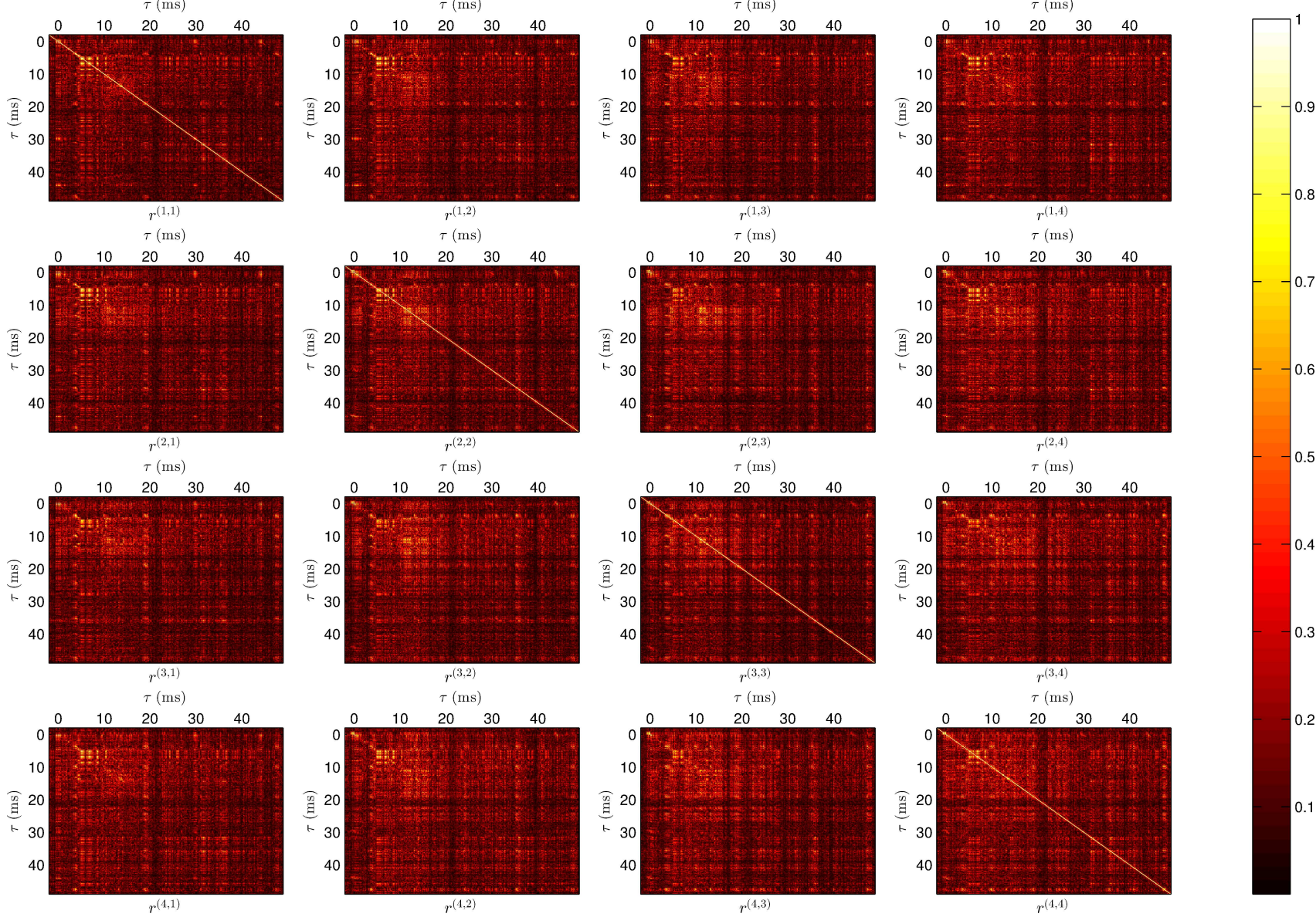

Fig. 3. Sample correlation matrices. Matrix entries are defined as $\left[r^{(m, n)}\right]_{l p} \triangleq\left|\frac{\sum_{k=0}^{K-1} \tilde{h}_{l}^{(m)}(k) \tilde{h}_{p}^{*}(n)(k)}{\sqrt{\sum_{k=0}^{K-1}\left|\tilde{h}_{l}^{(m)}(k)\right|^{2} \sum_{k=0}^{K-1}\left|\tilde{h}_{p}^{(n)}(k)\right|^{2}} \mid}\right|$

correlations in Fig. 3 have been computed across time for a single observation, the correlations of Fig. 4 have been computed across realizations for a fixed time (time averages vs ensemble averages). ${ }^{2}$ The ensemble averages have been obtained by Monte-Carlo simulation with 1000 trials. Since $\tilde{h}_{l}^{(m)}(k)$ is assumed to be ergodic and that our replay method (approximately) keeps the original statistics, it can be checked that both figures are similar.

Replay-based simulation can be useful to measure all sorts of statistics relevant for system design and/or validation [2][5], [12], [13]. For the sake of generality, we do not focus on a specific communication system but present numerical results on the mutual information between the input and output of the SIMO channel. More precisely, for an input block of $N$ symbols, with $N>L$, the SIMO channel input-output relationship can be written as

$$
\mathbf{z}(k)=\underbrace{\left[\begin{array}{c}
\mathbf{H}_{1}(k) \\
\mathbf{H}_{2}(k) \\
\vdots \\
\mathbf{H}_{M}(k)
\end{array}\right]}_{\triangleq \mathbf{H}(k)} \mathbf{x}(k)+\mathbf{w}(k),
$$

${ }^{2}$ Note that since $\tilde{h}_{l}^{(m)}(k)$ is stationary, the ensemble average can be computed for any fixed time sample $k$. where $\mathbf{x}(k)=[x(k), x(k+1), \cdots, x(k+N-1)]^{T}$ is the $N \times 1$ input vector at time $k, \mathbf{z}(k)$ the $M N \times 1$ output vector and $w(k)$ is the $M N \times 1$ noise vector. The $N \times N$ sub-block matrix $\mathbf{H}_{m}(k)$ is defined as ${ }^{3}$

$$
\begin{aligned}
& \mathbf{H}_{m}(k) \triangleq \\
& {\left[\begin{array}{ccccc}
h_{0}^{(m)}(k) & & & & \\
h_{1}^{(m)}(k+1) & h_{0}^{(m)}(k+1) & & & \\
\vdots & & & 0 \\
h_{L-1}^{(m)}(k+L-1) & & \ddots & & \\
& \ddots & & & \\
0 & & h_{L-1}^{(m)}(k+N-1) & \ldots & h_{0}^{(m)}(k+N-1)
\end{array}\right]}
\end{aligned}
$$

We study the instantaneous mutual information (MI) of $\mathbf{z}(k)$ and $\mathbf{x}(k)$ (conditional on $\mathbf{H}$ ) that is defined as

$$
\begin{aligned}
\mathcal{I}(k) & \triangleq \mathbf{I}(\mathbf{z}(k) ; \mathbf{x}(k) \mid \mathbf{H}(k)) \\
& =\mathbf{h}(\mathbf{z}(k) \mid \mathbf{H}(k))-\mathbf{h}(\mathbf{z}(k) \mid \mathbf{x}(k), \mathbf{H}(k)),
\end{aligned}
$$

where $\mathrm{I}(\cdot)$ denotes mutual information and $\mathrm{h}(\cdot)$ relative entropy. Since it is a measure of dependence between $\mathbf{x}$ and $\mathbf{z}$,

${ }^{3}$ Note that (10) is valid only if the sampling frequency of the TVIR is same along the time and delay axes. 

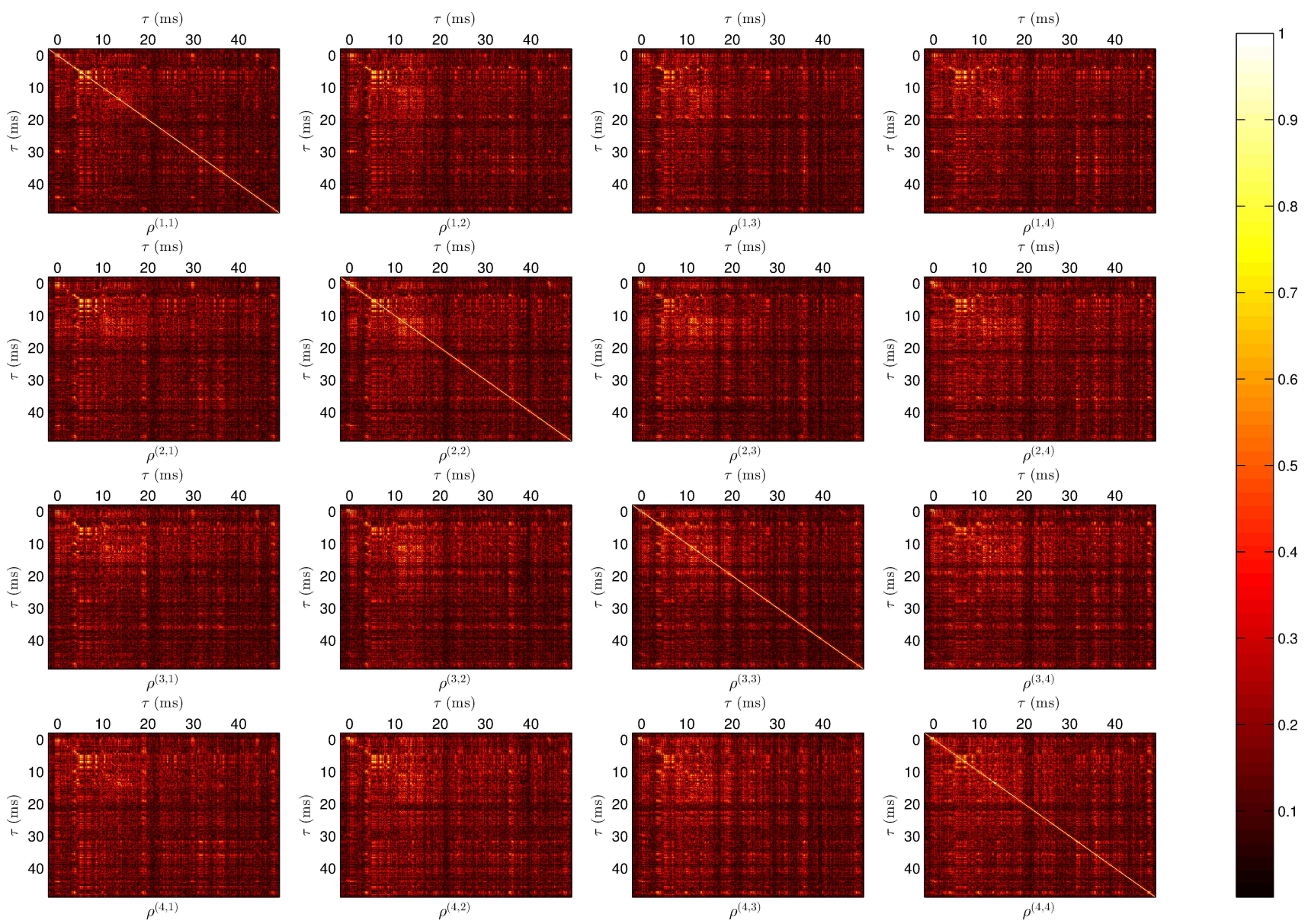

Fig. 4. Population correlation matrices. Matrix entries are defined as $\left[\rho^{(m, n)}\right]_{l p} \triangleq\left|\frac{\mathbb{E}_{\theta}\left\{y_{l}^{(m)}(k) y_{p}^{*}(n)(k) \mid \tilde{h}\right\}}{\sqrt{\mathbb{E}_{\theta}\left\{\left|y_{l}^{(m)}(k)\right|^{2} \mid \tilde{h}\right\} \mathbb{E}_{\theta}\left\{\left|y_{p}^{(n)}(k)\right|^{2} \mid \tilde{h}\right\}}}\right|$.

$\mathcal{I}(k)$ reflects the severity of the channel. We kept this MI time dependent since, according to (2), the first order statistics of $\mathbf{H}$ may vary over time. ${ }^{4}$ If we consider circularly symmetric Gaussian inputs as well as white Gaussian noise, the mutual information (12) is shown to satisfy

$$
\mathcal{I}(k)=\mathbb{E}_{\mathbf{H}}\left\{\log \operatorname{det}\left(\mathbf{I}_{M N}+\gamma \mathbf{H}(k) \mathbf{H}^{\dagger}(k)\right)\right\},
$$

where $\gamma$ is the signal-to-noise ratio (SNR).

Fig. 5 shows the time evolution of the MI in three different cases. The average SNR is set to $10 \mathrm{~dB}$ and the block size $N$ corresponds to a $200 \mathrm{~ms}$ time window. ${ }^{5}$ In the first case (dashdoted line), the mutual information is directly assessed on the measured channel without applying stochastic replay. Since ensemble averaging is not possible in this case, the expression of the plotted MI is simply $\log \operatorname{det}\left(\mathbf{I}_{M N}+\gamma \mathbf{H}(k) \mathbf{H}^{\dagger}(k)\right)$. The second case (black solid line) corresponds to (14) where the expectation $\mathbb{E}_{\mathbf{H}}\{\cdot\}$ is computed thanks to stochastic replay. This MI obviously fluctuates less than in the case of "direct" replay as it is averaged over 250 channel realizations.

\footnotetext{
${ }^{4}$ Note that the MI (12) is the not the channel capacity as perfect knowledge of $\mathbf{H}$ at reception cannot be assumed in most underwater acoustic contexts (see [12]-[14] for more details).

${ }^{5}$ Note that to account for frequency selectivity the block size $N$ must be greater than the number of taps $L$.
}

However, slow variations attributable to local fluctuations of channel energy are still visible. As shown in [5], with stochastic replay it is possible to deliberately distort the statistics of the experimental channel in order to meet some user-specified constraints. This capability is exploited in the third case (red solid line) where the impact of correlated scattering on the MI is assessed. More precisely, we have seen previously that the random components $\tilde{h}_{l}^{(n)}(k)$ are correlated across both taps and channels (see Fig. 3 and 4). Since correlation usually implies a loss of diversity, it may be interesting to quantify the impact of this correlation on the MI. This is rather easy with replay-based simulation since this is equivalent to generate a process $y$ with a diagonal covariance matrix that satisfies, for all $u$,

$$
\left[\boldsymbol{\Sigma}_{y}^{(m, n)}(u)\right]_{l p}=\left\{\begin{array}{cc}
{\left[\boldsymbol{\Sigma}_{\tilde{h}}^{(m, n)}(u)\right]_{l p}} & \text { for } l=p \text { and } m=n \\
0 & \text { otherwise. }
\end{array}\right.
$$

It can be shown that this property is satisfied (up to the scale factor $(K-|u|) / K)$ if the random phases defined in (8) are forced to be mutually independent for all $v \neq w, m \neq n$ and $l \neq p$. As visible in Fig. 5, decorrelating the channel random components results in a $5 \%$ increase of MI, which means that the MI loss attributable to correlated scattering is 


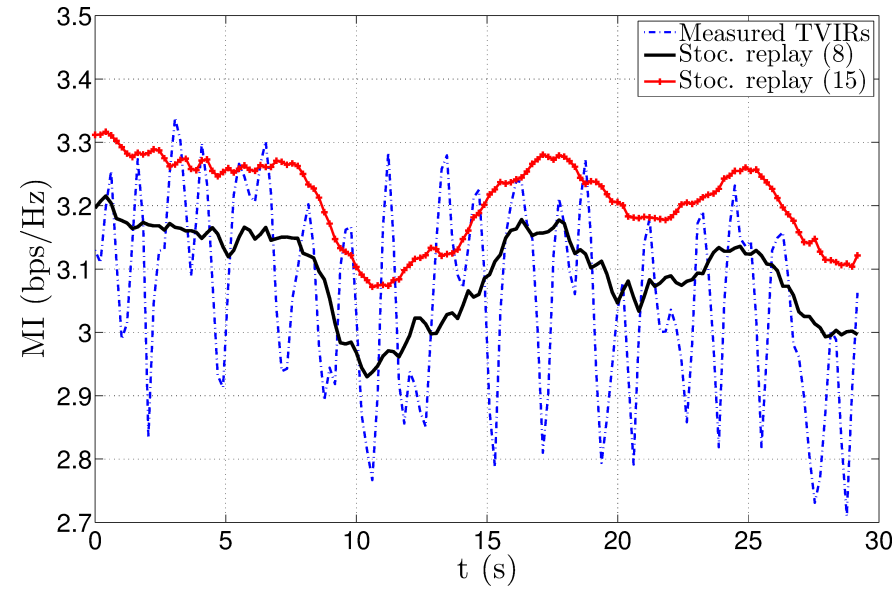

Fig. 5. Mutual information as function of a time. dash-doted line: direct replay, black solid line: stochastic replay as implemented in (8), red solid line: stochastic replay as implemented in (15). $\gamma=20, N=1000$.

rather negligible in our case.

\section{CONCLUSiOnS}

An extension of the simulator presented in [3] to SIMO configurations has been proposed. Through theoretical derivations and real-data analysis, it is shown that our stochastic replay strategy is able to preserve correlations of the measured TVIR jointly in time, delay and space. Our simulator has been used on real-data to quantify the mutual information loss attributable to inter-tap and spatial covariations. This loss is less than $5 \%$ for our dataset.

A great advantage of our approach is that it does not require an explicit estimation of correlations to generate new channel realizations. However, as noticed in Sec. II, a drawback is that it implicitly assumes negligible pseudocovariances. Such an assumption is valid if the covariances between the real and the imaginary parts of the channel taps exhibit some specific symmetry (see $[15$, p. 44]). A preliminary analysis of our dataset (not show here) seems to indicate that this assumption is reasonable, but this may not be the case for every UAC channels, especially in the ultrawideband case. Taking into account pseudocovariances in replay simulators is possible: it requires to work with composite taps whose real and imaginary parts are split up as done in [5] but at the expense of increased computational complexity.

\section{REFERENCES}

[1] R. Galvin and R.E.W. Coats, "A stochastic underwater acoustic channel model," in Proc. IEEE Oceans Conf., Sep. 1996.

[2] P. van Walree, P. Jenserud, and M. Smedsrud, "A Discrete-Time Channel Simulator Driven by Measured Scattering Functions," IEEE J. Sel. Areas Commun., vol. 26, no. 9, pp. 1628-1637, 2008.

[3] F.-X. Socheleau, C. Laot, and J.-M. Passerieux, "Stochastic Replay of non-WSSUS Underwater Acoustic Communication Channels Recorded at Sea," IEEE Trans. Signal Process., vol. 59, no. 10, pp. 4838-4849, 2011.

[4] R. Otnes, P. van Walree, and T. Jenserud, "Validation of Replay-Based Underwater Acoustic Communication Channel Simulation," IEEE J. Ocean. Eng., vol. 38, no. 4, pp. 689-700, 2013.

[5] F.-X. Socheleau, C. Laot, and J.-M. Passerieux, "Parametric ReplayBased Simulation of Underwater Acoustic Communication Channels," IEEE J. Ocean. Eng., 2015, To appear, available at https://sites.google. $\mathrm{com} / \mathrm{site} / \mathrm{fxsocheleau/publications.}$

[6] S. Basagni, C. Petrioli, R. Petroccia, and D. Spaccini, "Channel replaybased performance evaluation of protocols for underwater routing," in Oceans - St. John's, 2014, Sept 2014, pp. 1-7.

[7] M. Naderi, M. Pätzold, and A. G. Zaji, "The Design of MeasurementBased Underwater Acoustic Channel Simulators Using the INLSA Algorithm," in Proc. IEEE Oceans 2015, Genova, 2015.

[8] J. Cross, Jian Zhang, and Y.R. Zheng, "Impact of spatial correlation of fading channels on the performance of mimo underwater communication systems," in MILITARY COMMUNICATIONS CONFERENCE, 2011 MILCOM 2011, Nov 2011, pp. 430-434.

[9] A.G. Zajic, "Statistical space-time-frequency characterization of mimo shallow water acoustic channels," in OCEANS 2009, MTS/IEEE Biloxi Marine Technology for Our Future: Global and Local Challenges, Oct 2009, pp. 1-6.

[10] B. Tomasi, G. Zappa, K. McCoy, P. Casari, and M. Zorzi, "Experimental study of the space-time properties of acoustic channels for underwater communications," in OCEANS 2010 IEEE - Sydney, May 2010, pp. 1-9.

[11] F.-X. Socheleau, J.-M. Passerieux, and C. Laot, "Acoustic Modems Performance Assessment via Stochastic Replay of at Sea Recorded underwater Acoustic Communication Channels," in Proc. Underwater Acoustic Measurement: Technologies and Results, Jun. 2011.

[12] F.-X. Socheleau, M. Stojanovic, C. Laot, and J.-M. Passerieux, "Information-Theoretic Analysis of Underwater Acoustic OFDM Systems in Highly Dispersive Channels," Journal of Electrical and Computer Engineering, 2012, Article ID 716720.

[13] J.-M. Passerieux, F.-X. Socheleau, and C. Laot, "Achievable rates over doubly selective rician-fading channels under peak-power constraint," IEEE Trans. Wireless Commun., vol. 12, no. 2, pp. 586-594, February 2013.

[14] C. Polprasert, J.Ritcey, and M.Stojanovic, "Capacity of OFDM Systems over Fading Underwater Acoustic Channels," IEEE J. Ocean. Eng., vol. 36, no. 4, pp. 514-524, Oct. 2011.

[15] Gordon L. Stuber, Principles of Mobile Communication, Kluwer Academic Publishers, Norwell, MA, USA, 2nd edition, 1996. 